\title{
Total antioxidant intake and prostate cancer in the Cancer of the Prostate in Sweden (CAPS) study. A case control study
}

Kjell M. Russnes ${ }^{1,3^{*}}$ (D) Elisabeth Möller ${ }^{2}$, Kathryn M. Wilson ${ }^{4,5}$, Monica Carlsen ${ }^{3}$, Rune Blomhoff ${ }^{1,3}$, Sigbjørn Smeland ${ }^{3}$, Hans-Olov Adami², Henrik Grönberg ${ }^{2}$, Lorelei A. Mucci ${ }^{4,5}$ and Katarina Bälter ${ }^{2}$

\begin{abstract}
Background: The total intake of dietary antioxidants may reduce prostate cancer risk but available data are sparse and the possible role of supplements unclear. We investigated the potential association between total and dietary antioxidant intake and prostate cancer in a Swedish population.

Methods: We used FFQ data from 1499 cases and 1112 controls in the population based case-control study Cancer of the Prostate in Sweden (CAPS). The ferric reducing antioxidant potential (FRAP) assay was used to assess the total antioxidant capacity (TAC) of diet and supplements. We calculated odds ratios (ORs) for the risk of prostate cancer across quintiles of antioxidant intake from all foods, from fruit and vegetables only, and from dietary supplements using unconditional logistic regression.

Results: Coffee comprised $62 \%$ of the dietary antioxidant intake, tea $4 \%$, berries $4 \%$, chocolate $2 \%$, and boiled potatoes $2 \%$. In total $19 \%$ and $13 \%$ of the population took multivitamins and supplemental Vitamin C respectively, on a regular basis. Antioxidant intake from all foods and from fruits and vegetables separately measured by the FRAP assay was not associated with prostate cancer risk. For antioxidant intake from supplements we found a positive association with total, advanced, localized, high grade and low grade prostate cancer in those above median supplemental TAC intake of users compared to non-users (Adjusted ORs for total prostate cancer: 1. 37, $95 \%$ Cl 1.08-1.73, advanced: 1.51, 95 \% Cl 1.11-2.06, localized: 1.36. $95 \%$ Cl 1.06-1.76, high grade 1.60, $95 \%$ Cl 1.06-2.40, low grade 1.36, $95 \%$ Cl 1.03-1.81). A high intake of coffee ( $\geq 6$ cups/day) was associated with a possible risk reduction of fatal and significantly with reduced risk for high grade prostate cancer, adjusted OR: 0.45 (95\% Cl: 0.22-0.90), whereas a high intake of chocolate was positively associated with risk of total, advanced, localized and low grade disease (adjusted OR for total: 1.43, $95 \%$ Cl 1.12-1.82, advanced: 1.40, $95 \%$ Cl 1.01-1.96, localized: 1.43, $95 \%$ Cl 1.08-1.88, low-grade: 1.41, $95 \%$ Cl 1.03-1.93).
\end{abstract}

Conclusions: Total antioxidant intake from diet was not associated with prostate cancer risk. Supplement use may be associated with greater risk of disease.

Keywords: Antioxidants, Prostate cancer, Coffee, Dietary supplement

\footnotetext{
* Correspondence: k.m.russnes@medisin.uio.no

'Oslo University Hospital, Clinic of Cancer, Surgery and Transplantation,

Montebello 0380, Oslo, Norway

${ }^{3}$ Department of Nutrition, Institute for Basic Medical Sciences, University of

Oslo, Sognsvannsveien 9, 0372 Oslo, Norway

Full list of author information is available at the end of the article
} 


\section{Background}

The potential cancer protective effect of diets rich in antioxidants has been extensively studied in relation to different cancer sites. Most observational studies and intervention, have examined the effect of either single or a few antioxidant compounds, or a combined score of redox active compounds, but no consistent protective effect of antioxidant intake on prostate cancer has been shown [1-5].

Because naturally occurring antioxidants in, for example, fruits and vegetables work in a network of redox active compounds, it could be more informative and potentially reduce confounding by other effects of redox active compounds to study the total intake of antioxidants. Several methods have been developed to quantify total dietary antioxidant content [6]. We used the Ferric-reducing ability of plasma (FRAP) assay, a fast, easy to use, and reproducible method to assess total antioxidant content (TAC) in foods, beverages and supplements [7]. We recently reported that total antioxidant intake from diet reduced risk of total, lethal and advanced prostate cancer in the Health Professionals Follow-up Study [8]. We found a weak protective effect of total antioxidant intake from diet, as well as for coffee, one of the largest contributors to antioxidant intake. On the other hand, total antioxidant intake from supplements increased risk for lethal and advanced prostate cancer $[6,8]$.

In the current study, we examined the association between total antioxidant capacity (TAC) from diet and supplements and prostate cancer in a large population based case-control study of prostate cancer in Sweden. This study points out some differences in antioxidant intake between a Scandinavian and a US population, whilst the large number of cases allowed analyses of subgroups of prostate cancer.

\section{Methods}

\section{Study population}

The Cancer of the Prostate in Sweden (CAPS) study is a population-based case-control study of prostate cancer as described previously $[9,10]$. Cases from four of the six regional cancer registries in Sweden were recruited through treating physicians in 2001 and 2002. After approval from patients to participate, they were mailed a letter including consent form and included in the study when they filled out a self-administered questionnaire about lifestyle factors (including diet) and family history or donated blood samples which were returned to the study administration. The cancer was histopathologically or cytologically verified. Disease-related information, such as TNM (tumor, node, metastasis) status, clinical stage, Gleason score, and serum prostate-specific antigen (PSA) level at diagnosis was obtained from the National Prostate Cancer Registry and was available for $95 \%$ of the cases. Controls were randomly selected from the
Swedish population registry, identified by personal identification number, and frequency-matched to cases by age in 5-year age categories and by region of residence of the cases. When controls had been identified, they were contacted by mail, receiving a letter describing the study. A few weeks later they received the same letter with self-administered questionnaire and equipment for blood sampling as the cases. Linking control subjects with the National Cancer Registry identified potential control subjects with previous prostate cancer history. who were excluded. Of 1895 invited prostate cancer cases, 1499 (79 \%) completed the detailed baseline questionnaire about lifestyle and health. Average time between date of diagnosis and date when the questionnaire was sent was 5 months. Of the 1684 invited controls, 1130 (67\%) completed the questionnaire. All participants gave informed consent at the time of enrollment in the study. The ethics committees at Karolinska Institutet and Umeå University in Sweden approved the study.

\section{Dietary assessment}

Dietary data were collected as part of the questionnaire. All participants completed a validated, self-administered 109-item food frequency questionnaire (FFQ) that assessed the participants' frequency of consumption of foods and beverages over the previous 12 months. The questionnaire included ten additional questions on dietary supplements. This included information about frequency, dosage, type of supplement and duration of use. A shorter version of the FFQ had been validated earlier against weighed food records among women, and found correlations among the major contributors to TAC ranging from 0.32 to 0.71 [11]. Each FFQ item was assigned a FRAP value based on the average value of a limited number of variants of each food item, specified in the Antioxidant Food Table [7, 12] and foods specifically analyzed for this study (Additional file 1). For combined items such as berries, we used pre-estimated weighting of the individual sub-items (raspberries, blueberries etc.) to calculate a combined FRAP value. When an FFQ item did not have a specified FRAP value, the value was imputed based on knowledge of foods and beverages with similar antioxidant profiles. FRAP values were assigned to supplements in the same manner. All analyses of FRAP were performed at the Institute of Nutrition Research, University of Oslo.

To calculate each participant's total antioxidant capacity intake (TAC), the frequency of consumption of each item was multiplied by its FRAP value and summed across all items consumed. Three exposure variables for TAC intake were created: 1) Dietary TAC from all foods and beverages; 2) TAC from fruit and vegetables only; and 3) TAC from dietary supplements. We used data from the Swedish 
National Food Administration to calculate total energy intake and intake of nutrients based on the questionnaire data. TAC and nutrient intakes were energy-adjusted using the residual method $[8,13]$. We also examined the intake of the main contributors to TAC and their relation to prostate cancer risk.

\section{Statistical analysis}

Participants were divided into quintiles of TAC intake from all foods and from fruit and vegetables giving five levels of intake for these exposure variables. In addition we wanted to test whether the extreme low and high intakes of TAC were associated with disease and performed the calculation based on decile distribution of TAC intake. For TAC from supplements, the non-users comprised the referent group, and the users were categorized at the median intake, giving three levels of intake. This was due to the relatively large number of non-supplement users in this population. We used unconditional logistic regression models with indicator variables for each level of TAC intake, and for each intake category of the main contributors to TAC. Age group and region, matching factors in the study, were included in all models. The fully adjusted models also included: smoking status (never, former, current), BMI (<20, 20-22.5, 22.5-<25, 25-<27.5, 27.5$<30,>30 \mathrm{~kg} / \mathrm{m}^{2}$ ), education (0-9 years, 10-12 years, $13+$ yrs), and total energy intake (quartiles). Other potential confounders were tested in the model, including: civil/ marital status, employment status, family history of prostate cancer, physical activity, as well as intake of alphalinolenic acid, vitamin-D, calcium, phytoestrogens, red meat, fish and dairy products. None of these were included in the final models as they had no or little effect on the effect estimates or precision.

To test for dose-response trends across the levels of TAC exposures and categories of TAC contributors, we modeled all exposure variables as continuous variables using the median intake in each quintile, decile or category of intake. Analyses of the major contributors to dietary TAC (coffee, tea, berries, chocolate and boiled potatoes) were mutually adjusted, in addition to including the factors in the original TAC model, as well as adjusting for zinc and calcium intake. Similarly, the models for major contributors to supplemental TAC (Vitamin C supplements and multivitamins) were mutually adjusted, and included the same factors as the models for major contributors to dietary TAC. The analysis of intake of TAC and prostate cancer were also performed in subgroups as never smokers and ever smokers.

Advanced prostate cancer was defined as cancer with capsule penetration or seminal vesicle infiltration (T3), invasion of adjacent organs (T4), metastasis to lymph nodes $(\mathrm{N}+)$ or distant organs (M1) at the time of diagnosis, or prostate cancer death during follow-up through June 2009.
Fatal cases, a subgroup of advanced cases, were defined as participants that died from the disease during follow-up. Localized cases were those with T1 and T2 tumors and no metastases (N0/M0) at the time of diagnosis. Since advanced and lethal disease cases would include cases that died during follow-up, the categories localized and advanced are not mutually exclusive, and therefore the sum of advanced and localized exceeding the total number of cases. High grade cases included those with Gleason score 8-10, and low grade cases included cases with Gleason score 2-6. Gleason score 7 cases were not included in either highgrade or low-grade disease because of the heterogeneity of these tumors, and the different outcomes seen for Gleason $3+4$ compared to Gleason $4+3[6,8,14]$.

\section{Results}

\section{Descriptive data}

The main contributors to dietary TAC intake in the study population were coffee $(62 \%)$, tea (4\%), berries (4\%), chocolate $(2 \%)$ and boiled potatoes $(2 \%)$. Mean intake of TAC from diet was similar among controls and cases, 22.2 and $22.6 \mathrm{mmol} /$ day respectively, and TAC from fruit and vegetables was $3.5 \mathrm{mmol} /$ day among both cases and controls (Table 1). Among controls the mean intake of TAC from supplements was $0.6 \mathrm{mmol} /$ day compared to $0.8 \mathrm{mmol} /$ day among cases. We found no appreciable differences between controls and cases regarding BMI, smoking habits, and intake of energy, calcium and zinc. Multivitamin, supplemental vitamin $C$ and supplemental vitamin $\mathrm{E}$ use was more common among cases compared to controls and high grade cases used more multivitamins compared to controls. Intake of the major contributors to dietary TAC was similar across disease categories, except coffee and tea intakes that were slightly lower in high grade and fatal cases (Table 2).

\section{TAC and prostate cancer risk}

Dietary TAC and TAC from fruit and vegetables only were not significantly associated with prostate cancer risk (Table 3). To investigate whether the extreme high and low intakes of TAC were associated with all incident prostate cancer we calculated odds ratios based on decile distribution for the exposure variables dietary TAC $(\mathrm{OR}=$ 1.03, $95 \%$ CI $0.72-1.48$ and TAC from fruit and vegetables $(\mathrm{OR}=0.88$, $95 \% \mathrm{CI} 0.61-1.26)$ respectively between lowest and highest decile of intake.

We performed a stratified analysis of TAC intake from diet and fruit and vegetables in ever smokers and never smokers. No appreciable differences were observed compared to the total population. For dietary TAC and TAC from fruit \& vegetables among ever smokers the odds ratios in the highest quintile compared to the lowest quintile was: 1.12, (95\% CI 0.87-1.65) and 0.95 (95\% CI 0.69-1.31) respectively. Furthermore among never 
Table 1 Age standardized study population characteristic by controls and cases in means (standard deviations) or percents, Cancer of the Prostate Sweden Study

\begin{tabular}{lll}
\hline & Controls & Cases \\
$\mathrm{N}$ & 1112 & 1489 \\
\hline FRAP diet (mmol/d) & $22.2(8.6)$ & $22.3(8.4)$ \\
FRAP fruit/vegs (mmol/d) & $3.5(1.8)$ & $3.5(1.7)$ \\
FRAP suppl. (mmol/d) & $0.6(1.2)$ & $0.8(1.4)$ \\
Age & $67.7(7.5)$ & $66.8(7.3)$ \\
BMl & $26(3)$ & $26(3)$ \\
Current smokers (\%) & 12 & 11 \\
Never smokers (\%) & 38 & 39 \\
0-9 yrs education (\%) & 46 & 46 \\
10-12 years education (\%) & 42 & 40 \\
13+ yrs education (\%) & 11 & 14 \\
Energy kcal/d) & $2218(655)$ & $2283(646)$ \\
Coffee (cups/day) & $3.1(2.0)$ & $3.1(1.9)$ \\
Tea (cups/day) & $0.6(1.0)$ & $0.7(1.0)$ \\
Chocolate (serv./d) & $0.2(0.2)$ & $0.2(0.3)$ \\
Berries (serv./d) & $0.2(0.2)$ & $0.2(0.2)$ \\
Boiled potatoes (serv./d) & $0.6(0.4)$ & $0.6(0.4)$ \\
Multivitamin use (\%) & 16 & 22 \\
Vitamin C suppl. use (\%) & 12 & 15 \\
Vitamin E suppl. use (\%) & 3 & $11.6(1.9)$ \\
Calcium (mg/d) & $1197(363)$ & $89(360)$ \\
Zinc (mg/d) & $11.7(1.8)$ & \\
PSA at diagnosis & & 1590 \\
\hline & & \\
\hline
\end{tabular}

smokers the odds ratios in the highest quintile compared with the lowest was for dietary TAC 0.69, (95 \% CI $0.45-1.07)$ and TAC from fruit \& vegetables 0.96 , (95\% CI $0.63-1.48$ ) respectively.

Supplement use was more common among cases compared to controls, and consequently, we found a positive association between highest level of TAC intake from supplements and total, advanced, localized, high grade and low grade prostate cancer (total: $\mathrm{OR}=1.37,95 \% \mathrm{CI}$ 1.08-1.73, advanced: $1.51,95 \%$ CI 1.11-2.06, localized: 1.36 , 95 \% CI 1.06-1.76, high grade: $1.60,95$ \% CI $1.06-$ 2.40, low grade: $1.36,95 \%$ CI 1.03-1.81).

The major contributors to TAC derived from supplements were multivitamins and vitamin $\mathrm{C}$. We observed a positive association with use of the highest doses of multivitamins and total $(\mathrm{OR}=1.27,95 \% \mathrm{CI}: 1.01-1.60)$ and high grade prostate cancer risk (1.93, $95 \%$ CI: 1.32-2.81) (Table 4), whereas no association was seen for vitamin C supplements. To investigate if the findings for multivitamin use could be the result of reverse causation, (i.e., men began taking supplements as a result of feeling poorly due to disease prior to diagnosis), we took into account the duration of supplement use (vitamin $\mathrm{C}$ and multivitamins) and divided subjects into four groups: Never users, shortterm users ( $0-2$ years), intermediate-term users (2-5 years) and long-term users ( $>5$ years). We found a positive association for longer duration of multivitamin use and risk of advanced $(\mathrm{OR}=1.56,95 \% \mathrm{CI} 1.02-2.40)$ and highgrade prostate cancer $(2.00,95 \%$ CI $1.20-3.36)$ respectively for long-term users versus never users. For vitamin C use, we found a significantly increased risk of total, advanced and fatal prostate cancer among long-term compared to never users (total: $\mathrm{OR}=1.32,95 \%$ CI 1.04-1.66, advanced 1.52, $95 \%$ CI 1.03-2.23, fatal 1.23, $95 \% \mathrm{CI}$ $1.06-2.02)$.

\section{Components of dietary TAC and prostate cancer}

For the main contributors to dietary TAC intake there were some notable associations with prostate cancer (Table 4). As previously published for this population, coffee intake was inversely associated with risk of fatal and high grade prostate cancer [15]. Following mutual adjustment for the other contributors to TAC we found an inverse association between coffee intake and fatal and high grade prostate cancer with $45-55 \%$ lower OR for fatal and high grade prostate cancer, comparing the highest category of intake ( $\geq 6$ cups/day) to those in the lowest category of intake. For tea intake we observed a similar association, although weaker and based on fewer participants with high intakes. For berry intake we saw no significant inverse association. For chocolate intake, there was a positive association with total, advanced, localized and low grade prostate cancer (total $\mathrm{OR}=1.43$, 95 \% CI 1.12-1.82, advanced 1.40, 95 \% CI 1.01-1.96, localized 1.43, 95 \% CI 1.08-1.88, and low grade 1.41, $95 \%$ CI 1.03-1.93). For boiled potatoes, there was no significant trend across categories of intake.

\section{Discussion}

In this large Swedish population-based study, we found no convincing association between TAC from all foods or from fruit and vegetables only and overall prostate cancer. Other studies examining the risk of prostate cancer with a combined score of antioxidants and pro oxidants, also reported null results $[1,16]$. The assessment of the total dietary antioxidant capacity captures both all known and unknown antioxidants, which should be more informative than analyses of single antioxidants. Studies of total antioxidant intake utilizing the FRAP assay have been negative for colorectal and endometrial cancer. But for gastric cancer, an inverse association has been documented between high intake of antioxidants from plant foods assessed by the FRAP assay and disease risk [17-20].

One possible reason for the lack of overall association for TAC intake from diet and prostate cancer in our 
Table 2 Age standardized study population characteristic by subtypes of cases in means (standard deviations) or percents, Cancer of the Prostate Sweden Study

\begin{tabular}{|c|c|c|c|c|c|}
\hline & Fatal & Advanced & Localized & High grade & Low grade \\
\hline $\mathrm{N}$ & 307 & 499 & 1020 & 231 & 693 \\
\hline FRAP diet $(\mathrm{mmol} / \mathrm{d})$ & $21.6(7.9)$ & $22.5(8.9)$ & $22.2(8.1)$ & $21.3(8.0)$ & $22.3(8.0)$ \\
\hline FRAP fruit/vegs (mmol/d) & $3.5(1.9)$ & $3.4(1.7)$ & $3.5(1.7)$ & $3.6(2.0)$ & $3.4(1.6)$ \\
\hline FRAP suppl. (mmol/d) & $0.7(1.2)$ & $0.8(1.4)$ & $0.9(1.4)$ & $0.9(1.3)$ & $0.8(1.4)$ \\
\hline Age & $67.0(7.3)$ & $68.6(7.4)$ & $66.0(7.1)$ & $68.3(7.4)$ & $65.4(7.0)$ \\
\hline BMl & $26(3)$ & $26(3)$ & $26(3)$ & $26(3)$ & $26(4)$ \\
\hline Current smokers (\%) & 14 & 13 & 10 & 12 & 10 \\
\hline Never smokers (\%) & 42 & 39 & 39 & 41 & 38 \\
\hline 0-9 years education (\%) & 53 & 51 & 44 & 48 & 44 \\
\hline 10-12 years education (\%) & 37 & 41 & 40 & 40 & 41 \\
\hline $13+$ yrs education (\%) & 10 & 8 & 17 & 13 & 16 \\
\hline Energy $\mathrm{kcal} / \mathrm{d}$ ) & $2363(701)$ & 2334(681) & $2270(630)$ & $2387(655)$ & $2234(624)$ \\
\hline Coffee (cups/day) & $3.1(1.8)$ & $3.2(2.0)$ & $3.1(1.9)$ & $3.0(1.9)$ & $3.1(1.8)$ \\
\hline Tea (cups/day) & $0.5(0.8)$ & $0.5(0.9)$ & $0.7(1.1)$ & $0.6(0.8)$ & $0.7(1.1)$ \\
\hline Chocolate (serv./d) & $0.2(0.3)$ & $0.2(0.3)$ & $0.2(0.2)$ & $0.2(0.2)$ & $0.2(0.2)$ \\
\hline Berries (serv./d) & $0.2(0.3)$ & $0.2(0.2)$ & $0.2(0.3)$ & $0.2(0.3)$ & $0.1(0.2)$ \\
\hline Boiled potatoes (serv./d) & $0.6(0.4$ & $0.6(0.4)$ & $0.6(0.4)$ & $0.6(0.3)$ & $0.6(0.4)$ \\
\hline Multivitamin use (\%) & 21 & 22 & 21 & 29 & 20 \\
\hline Vitamin C suppl. use (\%) & 12 & 15 & 15 & 16 & 14 \\
\hline Vitamin E suppl. use (\%) & 3 & 3 & 4 & 3 & 5 \\
\hline Calcium (mg/d) & $1241(406)$ & $1222(390)$ & $1188(363)$ & $1213(381)$ & $1193(363)$ \\
\hline Zinc $(\mathrm{mg} / \mathrm{d})$ & $11.5(1.9)$ & $11.5(1.9)$ & $11.6(1.9)$ & $11.6(1.9)$ & $11.7(1.8)$ \\
\hline PSA at diagnosis & $264(573)$ & $276(665)$ & $39(241)$ & $167(364)$ & $22(136)$ \\
\hline
\end{tabular}

Fatal prostate cancer: Death from prostate cancer through follow up. Advanced prostate cancer: M1, N1, T3 or T4 and death during follow up. Localized prostate cancer: $\mathrm{T} 1$ or T2 and N0/M0. High-grade prostate cancer: Gleason sum 8-10. Low-grade prostate cancer: Gleason sum 2-6

study may be that some of the main contributors to the antioxidant intake had opposite associations with prostate cancer risk. Coffee, chocolate and berries were among the five major contributors, but as chocolate intake was positively associated with total, advanced, localized and low grade prostate cancer risk, the intake of coffee and berries seemed to be inversely associated with some of the disease sub-types.

TAC from coffee was suggestively associated with reduced risk for fatal and high grade cancer, which is in line with other population based studies [6, 15, 21, 22]. Recently several meta analyses have shown an inverse association between coffee consumption and prostate cancer, especially fatal and high grade cancers [23-26]. However, the composition of the diet with respect to TAC intake was different in the CAPS study compared to the Health Professionals Follow-up Study (HPFS) in the U.S. In the former study coffee contributed to $62 \%$ of dietary TAC intake but only $28 \%$ in the latter. The coffee intake in Sweden is considerably higher than in the US. Swedish per capita coffee consumption is $7.14 \mathrm{~kg} /$ year compared to the
US with only $4.24 \mathrm{~kg} /$ year (2011) [27]. This may also reflect that overall total antioxidant intake in Sweden is somewhat higher compared with other populations: Among cases and controls in this study the daily average intake (energy adjusted) was $22.3 \mathrm{mmol} / \mathrm{d}$ and $22.2 \mathrm{mmol} /$ d respectively. In the US population Health Professionals Follow up Study, the daily average intake of TAC from diet was $10.8 \mathrm{mmol} / \mathrm{d}$ [8]. In an Italian Study population the mean levels of total antioxidant intake from diet was $11.45 \mathrm{mmol} / \mathrm{d}$ utilizing the FRAP assay, but the authors did not include coffee in their calculation which may have resulted in an underestimation of the overall intake [28]. Other studies have used different units to quantify the antioxidant intake, making comparisons difficult [29].

The proportion of patients diagnosed with a higher clinical stage (T3, T4, N1 or M1) in the CAPS study was almost twice the proportion in the HPFS (33\% vs. $16 \%$ ) probably due to more extensive PSA screening in the U.S.

The observed association with chocolate could be biologically plausible, because the high amount of carbohydrates in chocolate could stimulate IGF-1 production as 
Table 3 Odds ratios, OR (and $95 \%$ Cl) of prostate cancer by quintiles of TAC all foods, TAC from fruits and vegetables, Cancer of the Prostate Sweden

\begin{tabular}{|c|c|c|c|c|c|c|}
\hline FRAP food only & Q1 & Q2 & Q3 & Q4 & Q5 & p-t rend \\
\hline All prostate cancer & $294 / 226$ & $292 / 228$ & $301 / 220$ & $308 / 212$ & $294 / 226$ & \\
\hline Age and region adjusted OR & 1.00 & $1.02(0.80-1.31)$ & $1.08(0.84-1.39)$ & $1.11(0.87-1.43)$ & $1.02(0.79-1.31)$ & 0.76 \\
\hline Fully adjusted OR & 1.00 & $0.98(0.77-1.26)$ & $1.05(0.82-1.35)$ & $1.09(0.85-1.40)$ & $1.05(0.82-1.36)$ & 0.53 \\
\hline Lethal prostate cancer & $51 / 226$ & $49 / 228$ & $49 / 220$ & $42 / 212$ & $41 / 226$ & \\
\hline Age and region adjusted OR & 1.00 & $0.98(0.63-1.51)$ & $1.01(0.65-1.56)$ & $0.91(0.58-1.43)$ & $0.89(0.57-1.43)$ & 0.58 \\
\hline Fully adjusted OR & 1.00 & $0.98(0.63-1.53)$ & $0.98(0.63-1.53)$ & $0.92(0.58-1.45)$ & $0.87(0.55-1.39)$ & 0.52 \\
\hline Advanced prostate cancer & $94 / 226$ & $103 / 228$ & $95 / 220$ & $102 / 212$ & $105 / 226$ & \\
\hline Age and region adjusted OR & 1.00 & $1.11(0.78-1.55)$ & $1.04(0.74-1.47)$ & $1.20(0.85-1.68)$ & $1.23(0.88-1.73)$ & 0.20 \\
\hline Fully adjusted OR & 1.00 & $1.08(0.77-1.53)$ & $1.02(0.72-1.44)$ & $1.18(0.83-1.67)$ & $1.23(0.87-1.74)$ & 0.21 \\
\hline Localized prostate cancer & $201 / 226$ & $196 / 228$ & $216 / 220$ & $212 / 212$ & $195 / 226$ & \\
\hline Age and region adjusted OR & 1.00 & $1.01(0.77-1.33)$ & $1.16(0.89-1.53)$ & $1.11(0.84-1.46)$ & $0.96(0.73-1.26)$ & 0.90 \\
\hline Fully adjusted OR & 1.00 & $0.96(0.73-1.27)$ & $1.12(0.85-1.47)$ & $1.09(0.82-1.43)$ & $1.00(0.75-1.32)$ & 0.80 \\
\hline High-grade prostate cancer & $56 / 226$ & $39 / 228$ & $47 / 220$ & $49 / 212$ & $40 / 226$ & \\
\hline Age and region adjusted OR & 1.00 & $0.72(0.46-1.13)$ & $0.88(0.57-1.36)$ & $0.99(0.64-1.52)$ & $0.79(0.50-1.25)$ & 0.64 \\
\hline Fully adjusted OR & 1.00 & $0.68(0.43-1.07)$ & $0.83(0.53-1.28)$ & $0.95(0.61-1.48)$ & $0.78(0.49-1.24)$ & 0.63 \\
\hline Low-grade prostate cancer & $134 / 226$ & $139 / 228$ & $136 / 220$ & $146 / 212$ & $138 / 226$ & \\
\hline Age and region adjusted OR & 1.00 & $1.06(0.78-1.44)$ & $1.07(0.78-1.45)$ & $1.11(0.81-1.50)$ & $0.99(0.72-1.35)$ & 0.22 \\
\hline Fully adjusted OR & 1.00 & $1.02(0.75-1.39)$ & $1.03(0.76-1.41)$ & $1.09(0.80-1.49)$ & $1.04(0.76-1.42)$ & 0.74 \\
\hline FRAP fruit \& vegetables & Q1 & Q2 & Q3 & Q4 & Q5 & $\mathrm{p}$-trend \\
\hline All prostate cancer & $297 / 222$ & $277 / 243$ & $306 / 213$ & $311 / 209$ & $295 / 224$ & \\
\hline Age and region adjusted OR & 1.00 & $0.84(0.66-1.08)$ & $1.06(0.82-1.36)$ & $1.09(0.85-1.39)$ & $0.97(0.76-1.25)$ & 0.50 \\
\hline Fully adjusted OR & 1.00 & $0.83(0.64-1.07)$ & $1.04(0.80-1.34)$ & $1.07(0.83-1.38)$ & $0.94(0.73-1.22)$ & 0.82 \\
\hline Lethal prostate cancer & $53 / 222$ & $33 / 243$ & $52 / 213$ & $45 / 209$ & $48 / 224$ & \\
\hline Age and region adjusted OR & 1.00 & $0.57(0.36-0.92)$ & $1.01(0.65-1.54)$ & $0.91(0.58-1.41)$ & $0.91(0.59-1.41)$ & 0.74 \\
\hline Fully adjusted OR & 1.00 & $0.61(0.38-0.99)$ & $1.13(0.73-1.77)$ & $1.07(0.67-1.71)$ & $1.02(0.65-1.61)$ & 0.39 \\
\hline Advanced prostate cancer & $114 / 222$ & $77 / 243$ & $106 / 213$ & $98 / 209$ & $102 / 224$ & \\
\hline Age and region adjusted OR & 1.00 & $0.64(0.44-0.94)$ & $0.90(0.63-1.30)$ & $0.92(0.64-1.33)$ & $0.91(0.63-1.30)$ & 0.76 \\
\hline Fully adjusted OR & 1.00 & $0.65(0.46-0.93)$ & $1.01(0.72-1.41)$ & $1.01(0.71-1.42)$ & $0.96(0.68-1.35)$ & 0.49 \\
\hline Localized prostate cancer & 192/222 & $204 / 243$ & $205 / 213$ & $219 / 209$ & 199/224 & \\
\hline Age and region adjusted OR & 1.00 & $0.93(0.71-1.23)$ & $1.09(0.83-1.44)$ & $1.15(0.87-1.51)$ & $1.00(0.76-1.32)$ & 0.67 \\
\hline Fully adjusted OR & 1.00 & $0.90(0.68-1.18)$ & $1.04(0.79-1.38)$ & $1.07(0.81-1.42)$ & $0.94(0.71-1.25)$ & 0.95 \\
\hline High-grade prostate cancer & $53 / 222$ & $27 / 243$ & $46 / 213$ & $45 / 209$ & $58 / 224$ & \\
\hline Age and region adjusted $O R$ & 1.00 & $0.47(0.28-0.77)$ & $0.88(0.57-1.37)$ & $0.89(0.57-1.39)$ & $1.08(0.71-1.64)$ & 0.12 \\
\hline Fully adjusted OR & 1.00 & $0.49(0.30-0.82)$ & $0.93(0.59-1.46)$ & $0.99(0.62-1.57)$ & $1.13(0.73-1.76)$ & 0.08 \\
\hline Low-grade prostate cancer & $129 / 222$ & $149 / 243$ & $140 / 213$ & $150 / 209$ & $124 / 224$ & \\
\hline Age and region adjusted OR & 1.00 & $0.99(0.73-1.34)$ & $1.06(0.78-1.45)$ & $1.15(0.85-1.57)$ & $0.91(0.66-1.24)$ & 0.67 \\
\hline Fully adjusted OR & 1.00 & $0.94(0.69-1.28)$ & $1.01(0.73-1.38)$ & $1.09(0.80-1.50)$ & $0.85(0.62-1.18)$ & 0.46 \\
\hline
\end{tabular}

Fully adjusted models are adjusted for: age, region, smoking (never, former, current), BMI (categories), education (categories), and energy intake (categories)

a possible mechanism contributing to increased tumor growth and a more malignant behavior of the disease $[30,31]$. In one study, sucrose intake and disaccharides was positively associated with prostate cancer risk [32]. However two large prospective studies found no association between glycemic index and prostate cancer risk [33, 34]. On the other hand, dark chocolate is also high in flavonoids, a potent group of antioxidants [12], but there was no distinction between different types of chocolate in the CAPS questionnaire. The most likely 
Table 4 Odds ratios, OR (and $95 \%$ Cl) of prostate cancer by major contributors to TAC: coffee, tea, berries, chocolate, boiled potatoes, multivitamins and vitamin C supplements, Cancer of the Prostate Sweden

\begin{tabular}{|c|c|c|c|c|c|c|}
\hline Coffee & None & $<2$ cups/day & 2-3cups/day & 4-5cups/day & $\geq 6$ cups/day & p-trend \\
\hline All prostate cancer & $139 / 98$ & $150 / 121$ & $644 / 491$ & $413 / 295$ & 143/107 & \\
\hline Fully adj. OR & 1.00 & $0.88(0.61-1.26)$ & $0.93(0.69-1.26)$ & $1.03(0.75-1.42)$ & $0.95(0.64-1.40)$ & 0.87 \\
\hline Fatal prostate cancer & $14 / 44$ & $32 / 175$ & $103 / 491$ & $68 / 295$ & $15 / 107$ & \\
\hline Fully adj. OR & 1.00 & $0.58(0.31-1.07)$ & $0.73(0.45-1.18)$ & $0.87(0.52-1.44)$ & $0.56(0.29-1.08)$ & 0.51 \\
\hline Advanced prostate cancer & $47 / 121$ & $47 / 121$ & $207 / 491$ & $151 / 295$ & $53 / 107$ & \\
\hline Fully adj. OR & 1.00 & $0.90(0.54-1.52)$ & $0.92(0.60-1.41)$ & $1.09(0.70-1.72)$ & $1.00(0.58-1.71)$ & 0.65 \\
\hline Localized prostate cancer & $98 / 98$ & 103/121 & $452 / 491$ & $273 / 295$ & $94 / 107$ & \\
\hline Fully adj. OR & 1.00 & $0.86(0.57-1.28)$ & $0.98(0.70-1.36)$ & $1.04(0.72-1.48)$ & $0.93(0.60-1.44)$ & 0.86 \\
\hline High grade prostate cancer & $30 / 98$ & $22 / 121$ & $98 / 491$ & $62 / 295$ & 19/107 & \\
\hline Fully adj. OR & 1.00 & $0.53(0.28-1.01)$ & $0.55(0.33-0.92)$ & $0.59(0.24-1.01)$ & $0.45(0.22-0.90)$ & 0.05 \\
\hline Low grade prostate cancer & $55 / 98$ & $79 / 121$ & $313 / 491$ & $177 / 295$ & $69 / 107$ & \\
\hline Fully adj. OR & 1.00 & $1.22(0.77-1.94)$ & $1.22(0.82-1.80)$ & $1.21(0.79-1.84)$ & $1.24(0.76-2.05)$ & 0.60 \\
\hline Tea & None & $<1$ cup/day & $1-2$ cups/day & $>2$ cups/day & & \\
\hline All prostate cancer & $605 / 484$ & $370 / 262$ & $439 / 310$ & $75 / 56$ & & \\
\hline Fully adj. OR & 1.00 & $1.12(0.91-1.37)$ & $1.09(0.89-1.34)$ & $0.97(0.65-1.44)$ & & 0.98 \\
\hline Fatal prostate cancer & $142 / 484$ & $73 / 262$ & $83 / 310$ & $9 / 56$ & & \\
\hline Fully adj. OR & 1.00 & $0.95(0.66-1.36)$ & $0.90(0.64-1.26)$ & $0.60(0.28-1.30)$ & & 0.16 \\
\hline Advanced prostate cancer & $230 / 484$ & $131 / 262$ & $121 / 310$ & $17 / 56$ & & \\
\hline Fully adj. OR & 1.00 & $1.06(0.81-1.40)$ & $0.86(0.64-1.15)$ & $0.67(0.37-1.23)$ & & 0.12 \\
\hline Localized prostate cancer & $390 / 484$ & $251 / 262$ & $318 / 310$ & $61 / 56$ & & \\
\hline Fully adj. OR & 1.00 & $1.15(0.92-1.45)$ & $1.20(0.96-1.50)$ & $1.12(0.74-1.70)$ & & 0.38 \\
\hline High grade prostate cancer & $94 / 484$ & $65 / 262$ & $63 / 310$ & $9 / 18$ & & \\
\hline Fully adj. OR & 1.00 & $1.27(0.88-1.83)$ & $0.92(0.63-1.36)$ & $0.64(0.29(1.40)$ & & 0.17 \\
\hline Low grade prostate cancer & $265 / 484$ & $166 / 262$ & $221 / 310$ & $41 / 56$ & & \\
\hline Fully adj. OR & 1.00 & $1.12(0.86-1.45)$ & $1.25(0.97-1.62)$ & $1.12(0.70-1.81)$ & & 0.33 \\
\hline Berries & None & $1-3 /$ month & $>1-3 /$ month & & & \\
\hline All prostate cancer & $276 / 231$ & $739 / 482$ & $474 / 399$ & & & \\
\hline Fully adj. OR & 1.00 & $1.16(0.93-1.44)$ & $0.90(0.70-1.44)$ & & & 0.08 \\
\hline Fatal prostate cancer & $67 / 231$ & $129 / 482$ & $610 / 399$ & & & \\
\hline Fully adj. OR & 1.00 & $0.95(0.67-1.37)$ & $0.87(0.59-1.27)$ & & & 0.47 \\
\hline
\end{tabular}


Table 4 Odds ratios, OR (and $95 \%$ Cl) of prostate cancer by major contributors to TAC: coffee, tea, berries, chocolate, boiled potatoes, multivitamins and vitamin C supplements, Cancer of the Prostate Sweden (Continued)

\begin{tabular}{|c|c|c|c|c|}
\hline Advanced prostate cancer & $98 / 231$ & $236 / 482$ & $164 / 399$ & \\
\hline Fully adj. OR & 1.00 & $1.15(0.85-1.55)$ & $0.90(0.65-1.25)$ & 0.15 \\
\hline Localized prostate cancer & $186 / 321$ & $513 / 482$ & $321 / 399$ & \\
\hline Fully adj. OR & 1.00 & $1.16(0.91-1.49)$ & $0.89(0.68-1.16)$ & 0.11 \\
\hline High grade prostate cancer & $38 / 231$ & $104 / 482$ & $89 / 399$ & \\
\hline Fully adj. OR & 1.00 & $1.19(0.78-1.83)$ & $1.10(0.70-1.72)$ & 0.91 \\
\hline Low grade prostate cancer & $133 / 231$ & $370 / 482$ & 190/399 & \\
\hline Fully adj. OR & 1.00 & $1.17(0.89-1.53)$ & $0.75(0.56-1.02)$ & $<0.01$ \\
\hline Chocolate & None & $1-3 /$ month & $>1-3 /$ month & \\
\hline All prostate cancer & $232 / 258$ & $645 / 457$ & $612 / 397$ & \\
\hline Fully adj. OR & 1.00 & $1.38(1.09-1.73)$ & $1.43(1.12-1.82)$ & 0.03 \\
\hline Fatal prostate cancer & $59 / 258$ & $119 / 457$ & $129 / 397$ & \\
\hline Fully adj. OR & 1.00 & $1.26(0.86-1.85)$ & $1.47(0.99-2.20)$ & 0.09 \\
\hline Advanced prostate cancer & $93 / 258$ & $194 / 457$ & $212 / 397$ & \\
\hline Fully adj. OR & 1.00 & $1.19(0.86-1.63)$ & $1.40(1.01-1.96)$ & 0.06 \\
\hline Localized prostate cancer & $146 / 258$ & $463 / 457$ & $411 / 397$ & \\
\hline Fully adj. OR & 1.00 & $1.46(1.13-1.89)$ & $1.43(1.08-1.88)$ & 0.11 \\
\hline High grade prostate cancer & $42 / 258$ & $88 / 457$ & $101 / 397$ & \\
\hline Fully adj. OR & 1.00 & $1.04(0.67-1.60)$ & $1.20(0.77-1.88)$ & 0.33 \\
\hline Low grade prostate cancer & $102 / 258$ & $310 / 457$ & $281 / 397$ & \\
\hline Fully adj. OR & 1.00 & $1.38(1.03-1.85)$ & $1.41(1.03-1.93)$ & 0.09 \\
\hline Boiled potatoes & $0-4 \times / w$ & $4-6 \times / w$ & $\geq 1 \times /$ day & \\
\hline All prostate cancer & $266 / 195$ & $668 / 545$ & $555 / 372$ & \\
\hline Fully adj. OR & 1.00 & $0.92(0.73-1.16)$ & $1.12(0.87-1.42)$ & 0.21 \\
\hline Fatal prostate cancer & $42 / 195$ & $151 / 545$ & $114 / 372$ & \\
\hline Fully adj. OR & 1.00 & $1.27(0.85-1.90)$ & $1.31(0.86-2.01)$ & 0.26 \\
\hline Advanced prostate cancer & $86 / 195$ & $219 / 545$ & $194 / 372$ & \\
\hline Fully adj. OR & 1.00 & $0.88(0.64-1.20)$ & $1.07(0.77-1.49)$ & 0.45 \\
\hline Localized prostate cancer & 181/195 & $464 / 545$ & $375 / 372$ & \\
\hline Fully adj. OR & 1.00 & $1.00(0.79-1.29)$ & $1.20(0.92-1.57)$ & 0.13 \\
\hline High grade prostate cancer & $33 / 195$ & $107 / 545$ & $91 / 372$ & \\
\hline
\end{tabular}


Table 4 Odds ratios, OR (and $95 \%$ Cl) of prostate cancer by major contributors to TAC: coffee, tea, berries, chocolate, boiled potatoes, multivitamins and vitamin C supplements, Cancer of the Prostate Sweden (Continued)

\begin{tabular}{|c|c|c|c|c|}
\hline Fully adj. OR & 1.00 & $1.18(0.75-1.84)$ & $1.33(0.83-2.12)$ & 0.21 \\
\hline Low grade prostate cancer & $134 / 195$ & $107 / 545$ & $91 / 372$ & \\
\hline Fully adj. OR & 1.00 & $0.90(0.68-1.19)$ & $1.07(0.79-1.45)$ & 0.45 \\
\hline Multivitamins & None & $<7 /$ week & $\geq 7 /$ week & \\
\hline All prostate cancer & $1166 / 930$ & $69 / 32$ & $254 / 150$ & \\
\hline Fully adj. OR & 1.00 & $1.48(0.95-2.30)$ & $1.27(1.01-1.60)$ & 0.07 \\
\hline Fatal prostate cancer & $183 / 930$ & $12 / 32$ & $37 / 150$ & \\
\hline Fully adj. OR & 1.00 & $1.65(0.84-3.24)$ & $1.37(0.95-1.99)$ & 0.18 \\
\hline Advanced prostate cancer & $391 / 930$ & $24 / 32$ & $84 / 150$ & \\
\hline Fully adj. OR & 1.00 & $1.69(0.97-2.97)$ & $1.29(0.95-1.75)$ & 0.08 \\
\hline Localized prostate cancer & $803 / 930$ & $46 / 32$ & $171 / 150$ & \\
\hline Fully adj. OR & 1.00 & $1.34(0.83-2.17)$ & $1.21(0.94-1.55)$ & 0.18 \\
\hline High grade prostate cancer & $165 / 930$ & $14 / 32$ & $52 / 150$ & \\
\hline Fully adj. OR & 1.00 & $2.20(1.11-4.33)$ & $1.93(1.32-2.81)$ & 0.03 \\
\hline Low grade prostate cancer & $552 / 930$ & $25 / 32$ & $116 / 150$ & \\
\hline Fully adj. OR & 1.00 & $1.08(0.62-1.89)$ & $1.25(0.85-1.85)$ & 0.75 \\
\hline Vitamin C & None & $<7 /$ week & $\geq 7 /$ week & \\
\hline All prostate cancer & $1271 / 984$ & $106 / 62$ & $112 / 66$ & \\
\hline Fully adj. OR & 1.00 & $1.20(0.86-1.68)$ & $1.22(0.88-1.70)$ & 0.14 \\
\hline Fatal prostate cancer & 200/984 & $19 / 62$ & $13 / 66$ & \\
\hline Fully adj. OR & 1.00 & $1.27(0.75-2.16)$ & $0.86(0.47-1.55)$ & 0.19 \\
\hline Advanced prostate cancer & $423 / 984$ & $36 / 62$ & $40 / 66$ & \\
\hline Fully adj. OR & 1.00 & $1.32(0.85-2.06)$ & $1.47(0.96-2.25)$ & 0.14 \\
\hline Localized prostate cancer & $866 / 984$ & $78 / 62$ & $76 / 66$ & \\
\hline Fully adj. OR & 1.00 & $1.25(0.87-1.79)$ & $1.15(0.80-1.65)$ & 0.20 \\
\hline High grade prostate cancer & 195/984 & $21 / 62$ & $15 / 66$ & \\
\hline Fully adj. OR & 1.00 & $1.48(0.86-2,55)$ & $0.93(0.50-1.71)$ & 0.80 \\
\hline Low grade prostate cancer & $593 / 984$ & $43 / 62$ & $57 / 66$ & \\
\hline Fully adj. OR & 1.00 & $0.99(0.65-1.51)$ & $1.25(0.85-1.85)$ & 0.18 \\
\hline
\end{tabular}

Fully adjusted models are adjusted for: age, region, smoking (never, former, current), BMI (categories), education (categories), energy intake, calcium (quartiles), zinc(quartiles). Food models mutually adjusted for chocolate (categories), coffee (categories), berries (categories), tea (categories), boiled potatoes (categories). Supplement models mutually adjusted for vitamin C supplement intake and multivitamin intake 
explanation for this association, however is that it is a chance finding.

Because berries are a rich source of flavonoids, the trend indicating an inverse association with low-grade prostate cancer is plausible. Flavonoids are antioxidants that may inhibit the matrix metalloproteinase system (MMP) [35, 36], which is probably important in the invasive and metastatic process [37]. The $25 \%$ reduction of risk for low-grade prostate cancer observed for those consuming berries more than 1-3 times per month could possibly be due to this chemopreventive effect. This novel finding is interesting due to a plausible mechanism of action. If a protective effect from substances abundant in berries exists - it would most likely be weak and mostly effective in the indolent, low-grade cases whereas an effect in high-grade cases would not be expected. In the CAPS questionnaire intake of all berries were aggregated into one question. This makes it difficult to determine whether the association was related to specific berries/phytochemicals or confounded by healthy lifestyle related behaviors. Furthermore, when we analyzed TAC from fruit and vegetables, where berries comprised $23 \%$ of the TAC contribution, we observed no association with prostate cancer.

TAC derived from supplements appeared positively associated with all categories of prostate cancer, except fatal cancers. The risk estimates are in line with those previously found in the Health Professionals Follow-up Study [8]. However, we cannot rule out reverse causation, because men who experienced early symptoms of prostate cancer before diagnosis might have selfmedicated with supplements. High doses of antioxidants taken as supplements may also have pro-oxidant properties [38, 39], that promote survival of malignant cells [40]. Recently, it was also shown in animal models, that dietary antioxidants may interfere with endogenous antioxidants by affecting feedback mechanisms [41]. All these explanations fits well with the observation that most nutrients follow a non-linear, inverted U-shaped curve with respect to their physiological function, and hence both deficiencies and high levels may be associated with disease [42]. Possible explanations for the differences between this population and the HPFS may be that almost twice as many individuals were regular supplement users in the US study population compared to the participants in CAPS. The long follow up time with multiple exposure assessments and large numbers cases with lethal disease in the HPFS can also explain why this finding was more apparent in the HPFS, compared to the fatal disease studied in CAPS.

In the present study we also had the ability to look more closely at the major contributors to TAC from supplements. The associations were mainly accounted for by multivitamin use, in line with findings in some other studies [43, 44], although others reported null results $[45,46]$. However, in our study, both analyses on TAC from supplements and multivitamins must be interpreted with caution due to the limited number of supplement users and the possibility of reverse causation.

We are aware of other limitations in this study. Selection bias is a concern in case-control studies, as participating controls may be more health conscious than the general population. In our study, however, the groups were well balanced with regard to baseline characteristics. In addition, such selection bias would not explain our supplement results because dietary supplement use is associated with more health conscious behavior, Recall bias is another concern, because diet was assessed after the prostate cancer diagnosis which could give rise to differential misclassification of exposure. Reverse causation, especially in the assessment of supplement use as mentioned above, may explain the findings associated with TAC from supplements, since a proportion of the cases may have experienced disease-related symptoms such as fatigue and started using supplements due to this before the time of diagnosis. Multiple testing is always a concern when assessing many different outcomes. The lack of association between TAC from diet and prostate cancer risk could also in part be explained by the fact that, the FRAP assay only measure in vitro antioxidant activity and, does not take into account the diversity in bioavailability of different antioxidants and their in vivo metabolism.

There are several strengths to this study. The population-based design with a large number of controls and cases, and information on disease characteristics makes it possible to study not only total prostate cancer, but also sub-groups of the disease. The large amount of data collected, including information on diet and other lifestyle-related habits, makes it possible to adjust for many possible confounding factors. The measurement of the total antioxidant capacity in diet captures both all known and unknown antioxidants, which may strengthen the associations compared to examining only single antioxidants.

\section{Conclusion}

In conclusion, we found no association between of total antioxidant capacity and prostate cancer, either from the total diet or from fruit and vegetables. High intake of antioxidants from supplements was associated with increased risk for all subgroups of prostate cancer, except fatal cancer. This finding must be interpreted with caution due to the possibility for reverse causation. 


\section{Additional files}

Additional file 1: FRAP values. Description: Supplementary table of FRAP values from food, beverages and dietary supplements unique for the Scandinavian market, and not included in antioxidant food table. (DOCX $119 \mathrm{~kb})$

\section{Abbreviations}

CAPS, Cancer of the Prostate in Sweden; FFQ, food frequency questionnaire; FRAP: ferric reducing antioxidant potential; HPFS, Health Professionals Follow-up Study; OR, odds ratio; TAC, total antioxidant capacity

\section{Funding}

The Throne Holst Foundation and Radiumhosptalets legater (The Norwegian Radiumhospital Foundation) funded the work.

The funding sources had no influence on the study design, collection of data, analysis and interpretation of data; the preparation of the manuscript or the decision of submitting the manuscript for publication.

\section{Availability of data and materials}

The CAPS data is not publically available at this time. The data includes personal information about the participants cancer diagnosis etc. The consent given by the participants in 2001-02 only permitted the Principal Investigator to use the data.

\section{Authors' contributions}

KMR: Hypothesis generation, performed SAS programming, data analysis and drafted the manuscript. EM: Generated FRAP values from the FFQ data and revised manuscript for submission. KMW: Performed SAS programming, data analysis and revised manuscript for submission. MC: Performed FRAP assay of substances included in supplementary table and revising manuscript for submission. RB: Planning and coordinating FRAP assay and revising manuscript for submission. SS: Revising manuscript for submission. HOA: Hypothesis generation, study coordination of CAPS and revising manuscript for submission. HG: Hypothesis generation, study coordination of CAPS and revising manuscript for submission. LAM: Hypothesis generation, data analysis and revising manuscript for submission. KB: Hypothesis generation, coordination of study, data analysis and revising manuscript for submission.

\section{Competing interests}

The authors declare that they have no competing interests.

\section{Consent for publication}

Not applicable

\section{Ethics approval and consent to participate}

All participants gave informed consent at the time of enrollment in the study. The ethics committees at Karolinska Institutet and Umeå University in Sweden approved the study.

\section{Author details}

'Oslo University Hospital, Clinic of Cancer, Surgery and Transplantation, Montebello 0380, Oslo, Norway. ${ }^{2}$ Department of Medical Epidemiology and Biostatistics, Karolinska Institute, 17177 Stockholm, Sweden. ${ }^{3}$ Department of Nutrition, Institute for Basic Medical Sciences, University of Oslo, Sognsvannsveien 9, 0372 Oslo, Norway. ${ }^{4}$ Department of Epidemiology, Harvard School of Public Health, 677 Huntington Avenue, Boston, MA 02115 , USA. ${ }^{5}$ Channing Laboratory, 181 Longwood Ave, Boston, MA 02115, USA.

\section{Received: 28 December 2015 Accepted: 4 July 2016}

\section{Published online: 11 July 2016}

\section{References}

1. Agalliu I, Kirsh VA, Kreiger N, Soskolne CL, Rohan TE. Oxidative balance score and risk of prostate cancer: results from a case-cohort study. Cancer Epidemiol. 2011;35:353-61.

2. Gaziano JM, Glynn RJ, Christen WG, Kurth T, Belanger C, MacFadyen J, Bubes V Manson JE, Sesso HD, Buring JE. Vitamins $E$ and $C$ in the prevention of prostate and total cancer in men: the Physicians' Health Study II randomized controlled trial. JAMA. 2009;301:52-62.
3. Heinonen OP, Albanes D, Virtamo J, Taylor PR, Huttunen JK, Hartman AM, Haapakoski J, Malila N, Rautalahti M, Ripatti S, Maenpaa H, Teerenhovi L, Koss L, Virolainen M, Edwards BK. Prostate cancer and supplementation with alpha-tocopherol and beta-carotene: incidence and mortality in a controlled trial. J Natl Cancer Inst. 1998;90:440-6.

4. Klein EA, Thompson IMJ, Tangen CM, Crowley JJ, Lucia MS, Goodman PJ, Minasian LM, Ford LG, Parnes HL, Gaziano JM, Karp DD, Lieber MM, Walther PJ, Klotz L, Parsons JK, Chin JL, Darke AK, Lippman SM, Goodman GE, Meyskens FU, Baker LH. Vitamin E and the risk of prostate cancer: the Selenium and Vitamin E Cancer Prevention Trial (SELECT). JAMA. 2011;306:1549-56.

5. Peters U, Littman AJ, Kristal AR, Patterson RE, Potter JD, White E. Vitamin E and selenium supplementation and risk of prostate cancer in the Vitamins and lifestyle (VITAL) study cohort. Cancer Causes Control. 2008;19:75-87.

6. Wilson KM, Kasperzyk JL, Rider JR, Kenfield S, van Dam RM, Stampfer MJ, Giovannucci E, Mucci LA. Coffee consumption and prostate cancer risk and progression in the health professionals follow-up study. J Natl Cancer Inst. 2011.

7. Benzie IF, Strain JJ. The ferric reducing ability of plasma (FRAP) as a measure of "antioxidant power": the FRAP assay. Anal Biochem. 1996;239:70-6.

8. Russnes KM, Wilson KM, Epstein MM, Kasperzyk JL, Stampfer MJ, Kenfield SA, Smeland S, Blomhoff R, Giovannucci EL, Willett WC, Mucci LA. Total antioxidant intake in relation to prostate cancer incidence in the Health Professionals Follow-Up Study. Int J Cancer. 2014;134:1156-65.

9. Hedelin M, Balter KA, Chang ET, Bellocco R, Klint A, Johansson JE, Wiklund F, Thellenberg-Karlsson C, Adami HO, Gronberg H. Dietary intake of phytoestrogens, estrogen receptor-beta polymorphisms and the risk of prostate cancer. Prostate. 2006;66:1512-20.

10. Lindmark F, Zheng SL, Wiklund F, Bensen J, Balter KA, Chang B, Hedelin M, Clark J, Stattin P, Meyers DA, Adami HO, Isaacs W, Gronberg H, Xu J. H6D polymorphism in macrophage-inhibitory cytokine-1 gene associated with prostate cancer. J Natl Cancer Inst. 2004;96:1248-54.

11. Khani BR, Ye W, Terry P, Wolk A. Reproducibility and validity of major dietary patterns among Swedish women assessed with a food-frequency questionnaire. J Nutr. 2004;134:1541-5.

12. Carlsen MH, Halvorsen BL, Holte K, Bohn SK, Dragland S, Sampson L, Willey C, Senoo H, Umezono Y, Sanada C, Barikmo I, Berhe N, Willett WC, Phillips KM, Jacobs DRJ, Blomhoff $R$. The total antioxidant content of more than 3100 foods, beverages, spices, herbs and supplements used worldwide. Nutr J. 2010;9:3

13. Willett WC, Howe GR, Kushi LH. Adjustment for total energy intake in epidemiologic studies. Am J Clin Nutr. 1997;65:1220S-8. discussion 1229S-1231S.

14. Stark JR, Perner S, Stampfer MJ, Sinnott JA, Finn S, Eisenstein AS, Ma J, Fiorentino M, Kurth T, Loda M, Giovannucci EL, Rubin MA, Mucci LA. Gleason score and lethal prostate cancer: does $3+4=4+3$ ? J Clin Oncol. 2009;27:3459-64.

15. Wilson KM, Balter K, Moller E, Adami HO, Andren O, Andersson SO, Gronberg H, Mucci LA. Coffee and risk of prostate cancer incidence and mortality in the Cancer of the Prostate in Sweden Study. Cancer Causes Control. 2013;24:1575-81.

16. Geybels MS, Verhage BA, van Schooten FJ, van den Brandt PA. Measures of combined antioxidant and pro-oxidant exposures and risk of overall and advanced stage prostate cancer. Ann Epidemiol. 2012:22:814-20.

17. Serafini M, Jakszyn P, Lujan-Barroso L, Agudo A, Bas Bueno-de-Mesquita $H$, van Duijnhoven FJ, Jenab $M$, Navarro $C$, Palli $D$, Boeing $H$, Wallstrom $P$, Regner S, Numans ME, Carneiro F, Boutron-Ruault MC, Clavel-Chapelon F, Morois S, Grioni S, Panico S, Tumino R, Sacerdote C, Ramon Quiros J, Molina-Montes E, Huerta Castano JM, Barricarte A, Amiano P, Khaw KT, Wareham N, Allen NE, Key TJ, et al. Dietary total antioxidant capacity and gastric cancer risk in the European prospective investigation into cancer and nutrition study. Int J Cancer. 2012;131:E544-54.

18. Mekary RA, Wu K, Giovannucci E, Sampson L, Fuchs C, Spiegelman D, Willett WC, Smith-Warner SA. Total antioxidant capacity intake and colorectal cancer risk in the Health Professionals Follow-up Study. Cancer Causes Control. 2010;21:1315-21.

19. Leufkens AM, van Duijnhoven FJ, Woudt SH, Siersema PD, Jenab M, Jansen EH, Pischon T, Tjonneland A, Olsen A, Overvad K, Boutron-Ruault MC, ClavelChapelon F, Morois S, Palli D, Pala V, Tumino R, Vineis P, Panico S, Kaaks R, Lukanova A, Boeing H, Aleksandrova K, Trichopoulou A, Trichopoulos D, Dilis V, Peeters PH, Skeie G, Gonzalez CA, Arguelles M, Sanchez MJ, et al. Biomarkers of oxidative stress and risk of developing colorectal cancer: a cohort-nested case-control study in the European Prospective Investigation Into Cancer and Nutrition. Am J Epidemiol. 2012;175:653-63. 
20. Gifkins D, Olson SH, Demissie K, Lu SE, Kong AN, Bandera EV. Total and individual antioxidant intake and endometrial cancer risk: results from a population-based case-control study in New Jersey. Cancer Causes Control. 2012:23:887-95

21. Geybels MS, Neuhouser ML, Wright JL, Stott-Miller M, Stanford JL. Coffee and tea consumption in relation to prostate cancer prognosis. Cancer Causes Control. 2013;24:941-948

22. Li Q, Kakizaki M, Sugawara Y, Tomata Y, Watanabe T, Nishino Y, Tsuji I. Coffee consumption and the risk of prostate cancer: the Ohsaki Cohort Study. Br J Cancer. 2013;108:2381-9.

23. Zhong S, Chen W, Yu X, Chen Z, Hu Q, Zhao J. Coffee consumption and risk of prostate cancer: an up-to-date meta-analysis. Eur J Clin Nutr. 2014;68:330-7.

24. Lu Y, Zhai L, Zeng J, Peng Q, Wang J, Deng Y, Xie L, Mo C, Yang S, Li S, Qin $X$. Coffee consumption and prostate cancer risk: an updated meta-analysis. Cancer Causes Control. 2014;25:591-604.

25. Cao S, Liu L, Yin X, Wang Y, Liu J, Lu Z. Coffee consumption and risk of prostate cancer: a meta-analysis of prospective cohort studies. Carcinogenesis. 2014;35:256-61.

26. Discacciati A, Orsini N, Andersson SO, Andren O, Johansson JE, Mantzoros CS, Wolk A. Coffee consumption and risk of localized, advanced and fatal prostate cancer: a population-based prospective study. Ann Oncol. 2013;24:1912-8.

27. International Coffee Organization. http://www.ico.org/new_historical. asp? section=Statistics.

28. La Vecchia C, Decarli A, Serafini M, Parpinel M, Bellocco R, Galeone C, Bosetti C, Zucchetto A, Polesel J, Lagiou P, Negri E, Rossi M. Dietary total antioxidant capacity and colorectal cancer: A large case-control study in Italy. Int J Cancer. 2013;133:1447-1452.

29. Pellegrini N1, Serafini M, Colombi B, Del Rio D, Salvatore S, Bianchi M, Brighenti F. Total antioxidant capacity of plant foods, beverages and oils consumed in Italy assessed by three different in vitro assays. J Nutr. 2003; 133(9):2812-9.

30. Hammarsten J, Hogstedt B. Hyperinsulinaemia: a prospective risk factor for lethal clinical prostate cancer. Eur J Cancer. 2005;41:2887-95.

31. Lehrer S, Diamond EJ, Stagger S, Stone NN, Stock RG. Serum insulin level, disease stage, prostate specific antigen (PSA) and Gleason score in prostate cancer. Br J Cancer. 2002:87:726-8.

32. Hu J, La Vecchia C, Gibbons L, Negri E, Mery L. Nutrients and risk of prostate cancer. Nutr Cancer. 2010:62:710-8.

33. Shikany JM, Flood AP, Kitahara CM, Hsing AW, Meyer TE, Willcox BJ, Redden DT, Ziegler RG. Dietary carbohydrate, glycemic index, glycemic load, and risk of prostate cancer in the Prostate, Lung, Colorectal, and Ovarian Cancer Screening Trial (PLCO) cohort. Cancer Causes Control. 2011;22:995-1002.

34. Nimptsch K, Kenfield S, Jensen MK, Stampfer MJ, Franz M, Sampson L, BrandMiller JC, Willett WC, Giovannucci E. Dietary glycemic index, glycemic load, insulin index, fiber and whole-grain intake in relation to risk of prostate cancer. Cancer Causes Control. 2011;22:51-61.

35. Matchett MD, MacKinnon SL, Sweeney MI, Gottschall-Pass KT, Hurta RA. Inhibition of matrix metalloproteinase activity in DU145 human prostate cancer cells by flavonoids from lowbush blueberry (Vaccinium angustifolium): possible roles for protein kinase $\mathrm{C}$ and mitogen-activated protein-kinase-mediated events. J Nutr Biochem. 2006;17:117-25.

36. Schmidt BM, Erdman JWJ, Lila MA. Differential effects of blueberry proanthocyanidins on androgen sensitive and insensitive human prostate cancer cell lines. Cancer Lett. 2006;231:240-6.

37. Kessenbrock K, Plaks V, Werb Z. Matrix metalloproteinases: regulators of the tumor microenvironment. Cell. 2010;141:52-67.

38. Schwartz JL. The dual roles of nutrients as antioxidants and prooxidants: their effects on tumor cell growth. J Nutr. 1996;126:1221S-7.

39. Burton GW, Ingold KU. beta-Carotene: an unusual type of lipid antioxidant. Science. 1984;224:569-73.

40. Schafer ZT, Grassian AR, Song L, Jiang Z, Gerhart-Hines Z, Irie HY, Gao S, Puigserver $P$, Brugge JS. Antioxidant and oncogene rescue of metabolic defects caused by loss of matrix attachment. Nature. 2009;461:109-13.

41. Sayin VI, Ibrahim MX, Larsson E, Nilsson JA, Lindahl P, Bergo MO. Antioxidants accelerate lung cancer progression in mice. Sci Transl Med. 2014:6:221ra15.

42. Morris MC, Tangney CC. A potential design flaw of randomized trials of vitamin supplements. JAMA. 2011;305:1348-9.

43. Lawson KA, Wright ME, Subar A, Mouw T, Hollenbeck A, Schatzkin A, Leitzmann MF. Multivitamin use and risk of prostate cancer in the National
Institutes of Health-AARP Diet and Health Study. J Natl Cancer Inst. 2007;99: 754-64

44. Stevens VL, McCullough ML, Diver WR, Rodriguez C, Jacobs EJ, Thun MJ, Calle EE. Use of multivitamins and prostate cancer mortality in a large cohort of US men. Cancer Causes Control. 2005;16:643-50.

45. Gaziano JM, Sesso HD, Christen WG, Bubes V, Smith JP, MacFadyen J, Schvartz M, Manson JE, Glynn RJ, Buring JE. Multivitamins in the prevention of cancer in men: the Physicians' Health Study II randomized controlled trial. JAMA. 2012;308:1871-80.

46. Hercberg S, Galan P, Preziosi P, Bertrais S, Mennen L, Malvy D, Roussel A-M, Favier A, Briançon S. The SU.VI.MAX Study: a randomized, placebocontrolled trial of the health effects of antioxidant vitamins and minerals. Arch Intern Med. 2004;164:2335-42.

\section{Submit your next manuscript to BioMed Central and we will help you at every step:}

- We accept pre-submission inquiries

- Our selector tool helps you to find the most relevant journal

- We provide round the clock customer support

- Convenient online submission

- Thorough peer review

- Inclusion in PubMed and all major indexing services

- Maximum visibility for your research

Submit your manuscript at www.biomedcentral.com/submit
Biomed Central 